Supporting Information

\title{
Emission Quenching and Recovery of Illuminated Perovskite Quantum Dots Due to lodide Ion Migration
}

\author{
Hua Wang ${ }^{1,2}$, Xiangtong Zhang ${ }^{2,3}$, Ning Sui ${ }^{1}$, Yue Hu${ }^{4}$, Vicki L. Colvin ${ }^{4}$, Xue Bai ${ }^{2}$ Yu Zhang ${ }^{2, *}$, \\ Andrey L. Rogach ${ }^{5, *}$, William W. Yu ${ }^{1,3, *}$
}

1 College of Material Science and Engineering, Qingdao University of Science and Technology, Qingdao 266042, China

2 State Key Laboratory on Integrated Optoelectronics, College of Electronic Science and Engineering, Jilin University, Changchun 130012, China

3 Department of Chemistry and Physics, Louisiana State University, Shreveport, LA 71115, US

4 Department of Chemistry, Brown University, Providence, RI 02912, US

5 Department of Materials Science and Engineering, and Centre for Functional Photonics (CFP), City University of Hong Kong, Kowloon, Hong Kong SAR, China

* Corresponding authors: andrey.rogach@cityu.edu.hk (A. L. Rogach), yuzhang@jlu.edu.cn (Y. Zhang), wyu6000@gmail.com (W.W. Yu) 


\section{Experimental Section}

Materials. $\mathrm{Cs}_{2} \mathrm{CO}_{3}$ (99.9\%) and $\mathrm{PbBr}_{2}$ (99.999\%) were purchased from Alfa Aesar; oleylamine (OAm, 70\%), 1-octadecene (ODE, 90\%) and oleic acid (OA, 90\%) were obtained from Sigma-Aldrich; $\mathrm{PbI}_{2}$ (99.995\%) was purchased from Beantown Chemical Corporation.

Preparation of Cs-oleate. $0.814 \mathrm{~g} \mathrm{Cs}_{2} \mathrm{CO}_{3}, 2.5 \mathrm{~mL} \mathrm{OA}$ and $30 \mathrm{~mL}$ ODE were mixed in a $100 \mathrm{~mL}$ 3-neck flask with vacuum and $\mathrm{N}_{2}$ connections. The system was degassed under vacuum for 10 $\min$, and heated to $120^{\circ} \mathrm{C}$ under vacuum for $1 \mathrm{~h}$. The mixture was further heated to $150^{\circ} \mathrm{C}$ under $\mathrm{N}_{2}$ and was kept for $2 \mathrm{~h}$ until a clear solution was obtained. Because Cs-oleate precipitates from ODE solution at room temperature, it was preheated to $120^{\circ} \mathrm{C}$ prior to the use.

Synthesis of $\mathrm{CsPbBr}_{1.2} \mathrm{I}_{1.8}$ PQDs. $0.105 \mathrm{~g} \mathrm{Pbl}_{2}, 0.052 \mathrm{~g} \mathrm{PbBr}_{2}, 1.0 \mathrm{~mL} \mathrm{OA}, 1.0 \mathrm{~mL} \mathrm{OAm}$ and $10 \mathrm{~mL}$ ODE were added into a $25 \mathrm{~mL}$ 3-neck flask. The flask was degassed under vacuum for $10 \mathrm{~min}$, kept under vacuum for $1 \mathrm{~h}$ at $120^{\circ} \mathrm{C}$, and connected to $\mathrm{N}_{2}$ gas. The temperature was raised to $160^{\circ} \mathrm{C}$ and $1.0 \mathrm{~mL}$ Cs-oleate/ODE was quickly injected. The reaction was terminated with an ice bath after $5 \mathrm{~s}$. The resulting solution was centrifuged to obtain a precipitate, which was dissolved in $2.0 \mathrm{~mL}$ hexane and centrifuged again. The resulting PQD solid was dispersed in 50 $\mathrm{mL}$ hexane, centrifuged to remove any possible aggregates, and re-dispersed in hexane, again. The concentration of the final PQDs in hexane was $5 \mathrm{mg} / \mathrm{mL}$.

Synthesis of $\mathrm{CsPbBr}_{2.4} \mathrm{I}_{0.6}$ and $\mathrm{CsPbl}_{3}$ PQDs. $0.0347 \mathrm{~g} \mathrm{Pbl}_{2}, 0110 \mathrm{~g} \mathrm{PbBr}_{2}, 1.0 \mathrm{~mL} \mathrm{OA}, 1.0 \mathrm{~mL} \mathrm{OAm}$ and $10 \mathrm{~mL}$ ODE were used to prepare $\mathrm{CsPbBr}_{2.4} \mathrm{I}_{0.6}$ PQDs. $0.174 \mathrm{~g} \mathrm{Pbl} 2,1.0 \mathrm{~mL} \mathrm{OA}, 1.0 \mathrm{~mL} \mathrm{OAm}$ and $10 \mathrm{~mL}$ ODE were used to prepare $\mathrm{CsPb}_{3}$ PQDs. Their syntheses were conducted in the same way as the synthesis of $\mathrm{CsPbBr}_{1.2} \mathrm{I}_{1.8}$ PQDs.

Characterization. UV-vis absorption spectra were collected on a Perkin Elmer Lambda 25 UV-VIS spectrometer. PL spectra were measured on a Horiba Jobin Yvon Fluorolog-3 spectrofluorometer. Transmission electron microscopy (TEM) images were obtained on a JEOL 
1400 TEM operated at 120kV. Fourier-transform infrared (FT-IR) spectra were recorded on a Thermo Scientific SMART FTIR spectrometer. X-ray photoelectron spectroscopy (XPS) was conducted on an ESCALAB250 instrument. XRD data were collected on a Bruker D8 Discovery 2D X-ray diffractometer. Time-resolved PL decays were measured on an Edinburgh Mini-tau spectrofluorometer. PL QYs were obtained on the same instrument using an integrating sphere.

DFT calculations. Calculations were performed within the framework of the density functional theory (DFT) as implemented in the Vienna Ab initio Software Package (VASP 5.3.5) code within the Perdew-Burke-Ernzerhof (PBE) generalized gradient approximation and the projected augmented wave (PAW) method. The cutoff energy for the plane-wave basis set was $400 \mathrm{eV}$. The Brillouin zone of the surface unit cell was sampled by Monkhorst-Pack (MP) grids, with a different k-point mesh for perovskite surface optimizations. The perovskite surfaces were determined by a $2 \times 2 \times 1$ Monkhorst-Pack grid. The convergence criterion for the electronic self-consistent iteration and force were set to $10^{-5} \mathrm{eV}$ and $0.01 \mathrm{eV} / \AA$, respectively. $\mathrm{A}$ vacuum layer of $35 \AA$ was introduced to avoid interactions between periodic images. The adsorption energy $\left(E_{a d s}\right)$ of the surface species was defined as $E_{a d s}=E_{\text {total }}-E_{\text {surface }}-E_{\text {species }}$, where $E_{\text {total }}$ represents the total energy of the adsorbed species on the surface, $E_{\text {surface }}$ is the energy of the empty perovskite surface, and $E_{\text {species }}$ is the energy of the species in the gas phase.

The free energies of adsorbates and transition states at temperature $\mathrm{T}$ were estimated according to the harmonic approximation, and the entropy was calculated as:

$$
S(T)=k_{\mathrm{B}} \sum_{i}^{\text {harm DOF }}\left[\frac{\varepsilon_{i}}{k_{\mathrm{B}} T\left(\mathrm{e}^{\varepsilon_{\mathrm{i}} / k_{\mathrm{B}} T}-1\right)}-\ln \left(1-\mathrm{e}^{-\varepsilon_{i} / k_{\mathrm{B}} T}\right)\right]
$$

where $k_{B}$ is the Boltzmann's constant and DOF is the number of harmonic energies $\left(\varepsilon_{i}\right)$ used in the summation denoted as the degree of freedom, which is generally $3 \mathrm{~N}$, where $\mathrm{N}$ is the number of atoms in the adsorbates or transition states. The free energies of gas phase species were calculated as:

$$
G_{\mathrm{g}}(T)=E_{\text {elec }}+E_{\mathrm{ZPE}}+\int C_{p} \mathrm{~d} T-T S(T)
$$


where $C_{p}$ is the gas phase heat capacity as a function of temperature derived from Shomate equations and the corresponding parameters in the equations were obtained from National Institute of Standards and Technology.

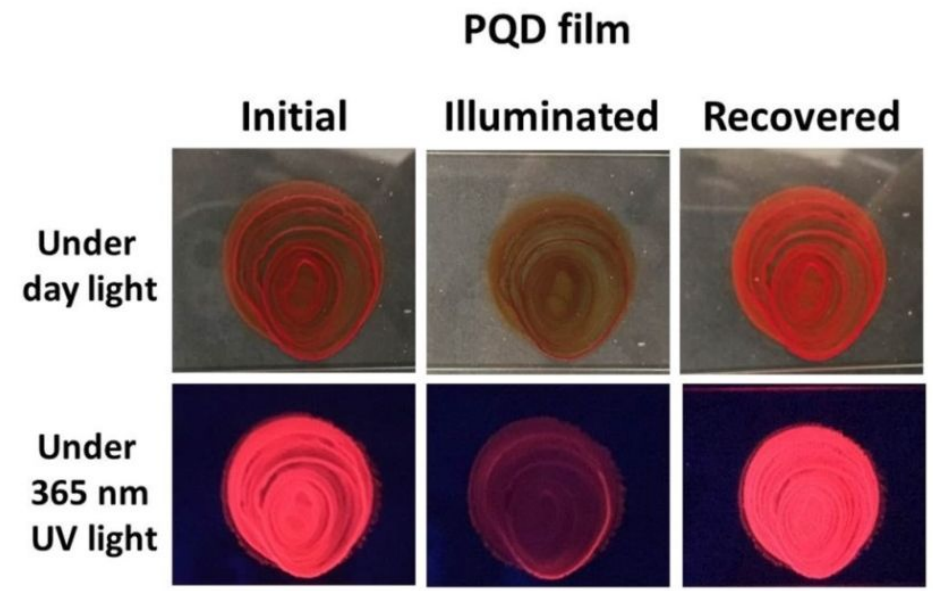

Figure S1. Photographs of the initial $\mathrm{CsPbBr}_{1.2} \mathrm{I}_{1.8} \mathrm{PQD}$ film, the same PQD film illuminated by blue LED light (460 nm, $100 \mathrm{~mW} / \mathrm{cm} 2$ ), and the one recovered in the dark. Photographs in the upper row were taken under daylight, while those in the bottom row - under $365 \mathrm{~nm}$ UV light excitation.

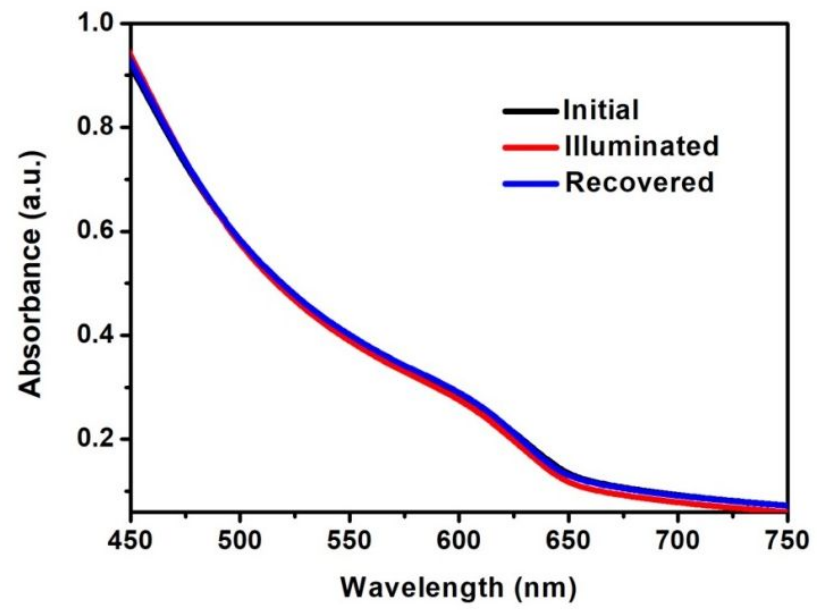

Figure S2. Absorption spectra of the initial, illuminated, and recovered $\mathrm{CsPbBr}_{1.2} \mathrm{I}_{1.8} \mathrm{PQDs}$ (in solution). 


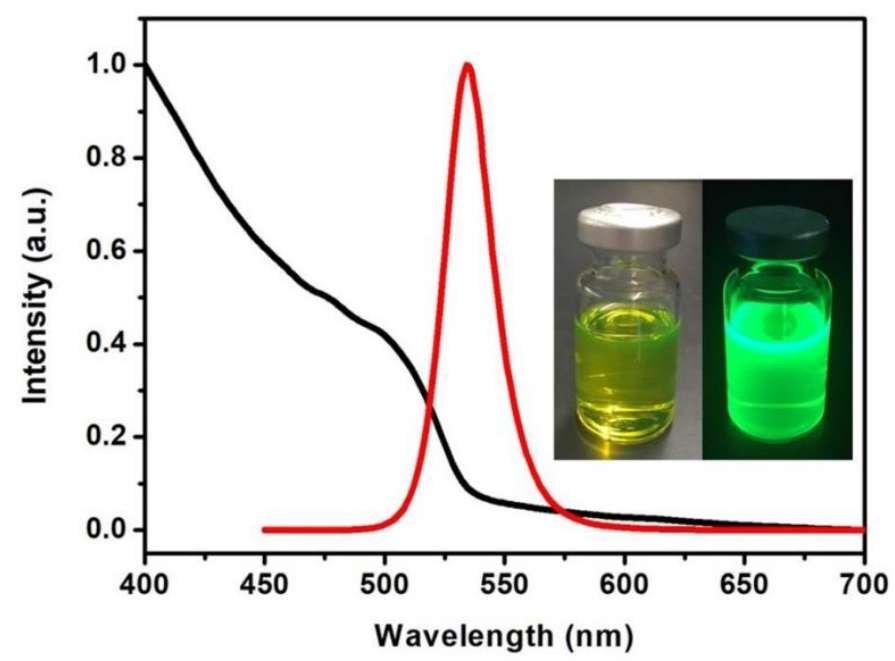

Figure S3. Absorption (black) and $\mathrm{PL}$ (red) spectra of $\mathrm{CsPbBr}_{2.4} \mathrm{I}_{0.6} \mathrm{PQDs}$ in solution, and photographs of the same PQDs in hexane taken under daylight (left) and under $365 \mathrm{~nm}$ UV light excitation (right).

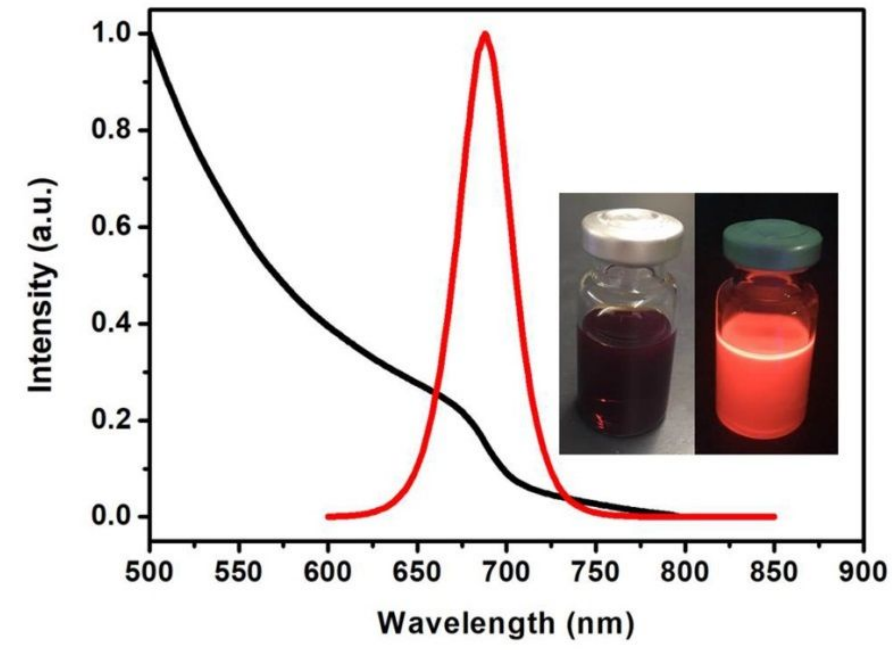

Figure S4. Absorption and PL spectra of $\mathrm{CsPbl}_{3} \mathrm{PQDs}$ in solution, and photographs of the same PQDs in hexane under daylight (left) and under $365 \mathrm{~nm}$ UV light excitation (right). 

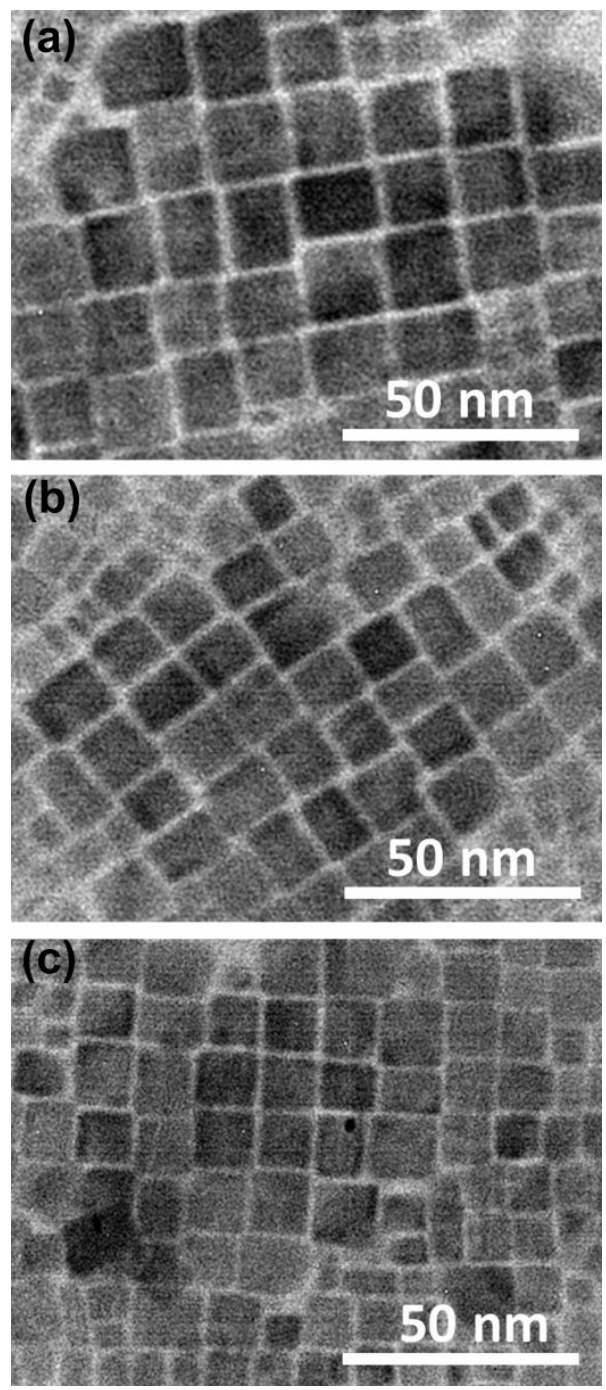

Figure S5. TEM images of the initial, illuminated, and recovered $\left.\mathrm{CsPbBr}_{1.2}\right|_{1.8} \mathrm{PQDs}$. 

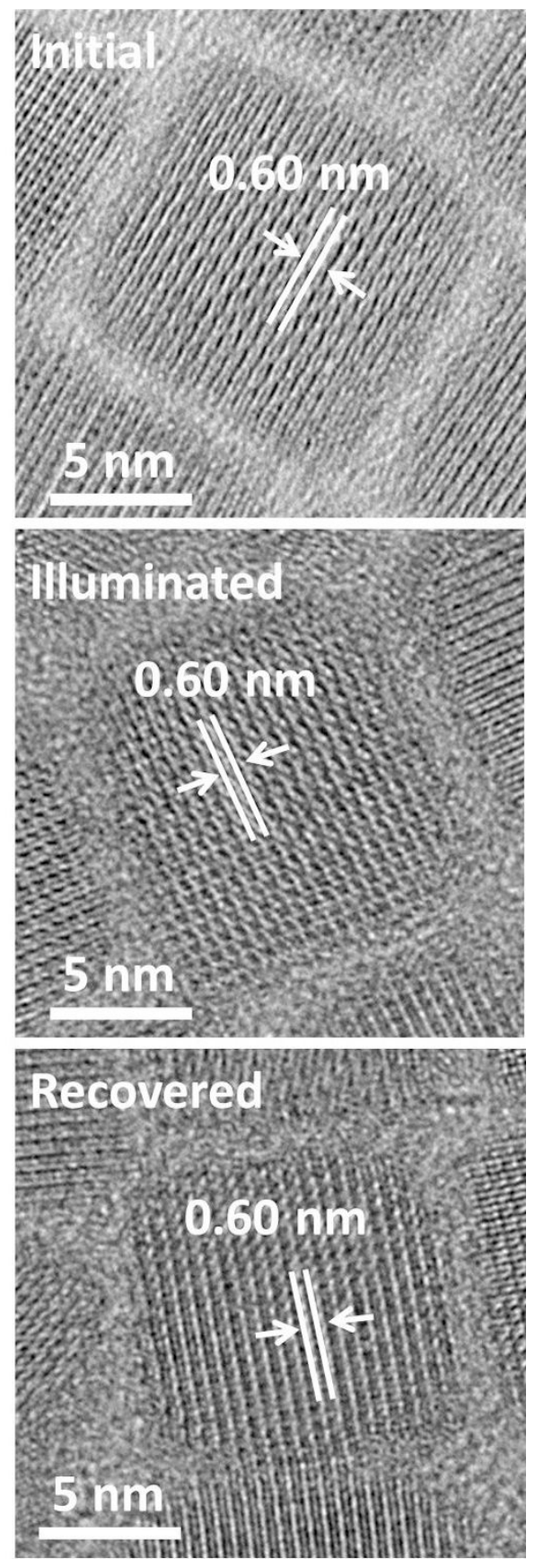

Figure S6. High-resolution TEM images of the initial, illuminated, and recovered $\mathrm{CsPbBr}_{1.2} \mathrm{I}_{1.8}$ PQDs. 


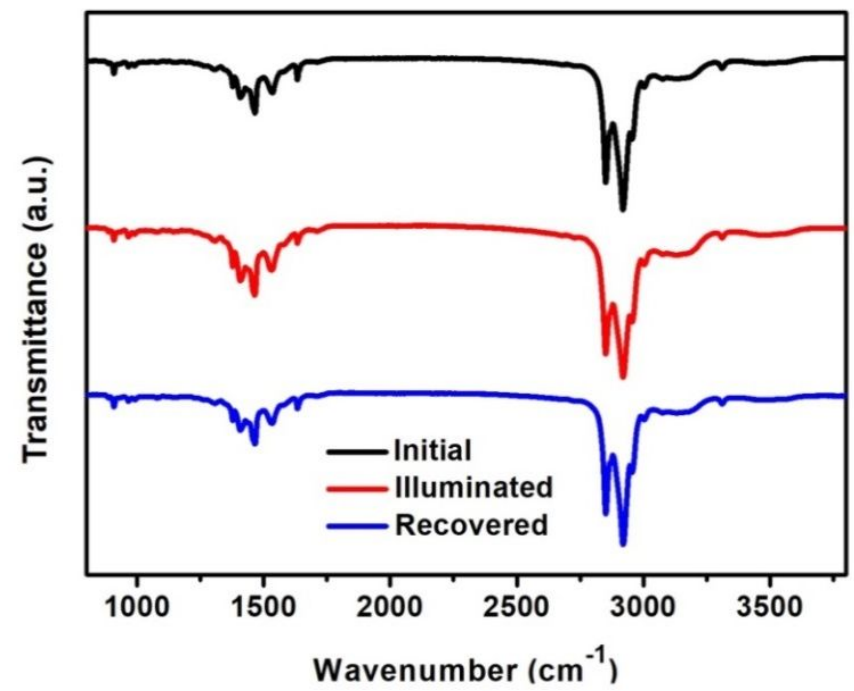

Figure S7. FT-IR spectra of the initial, illuminated, and recovered $\mathrm{CsPbBr}_{1.2} \mathrm{I}_{1.8} \mathrm{PQDs}$.

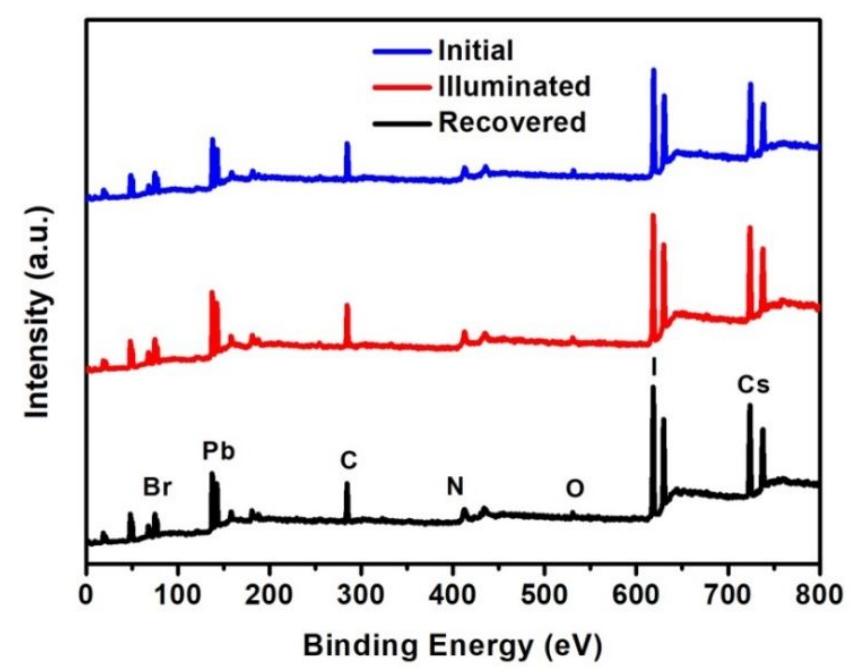

Figure S8. XPS spectra of the initial, illuminated, and recovered $\left.\mathrm{CsPbBr}_{1.2}\right|_{1.8}$ PQDs. 


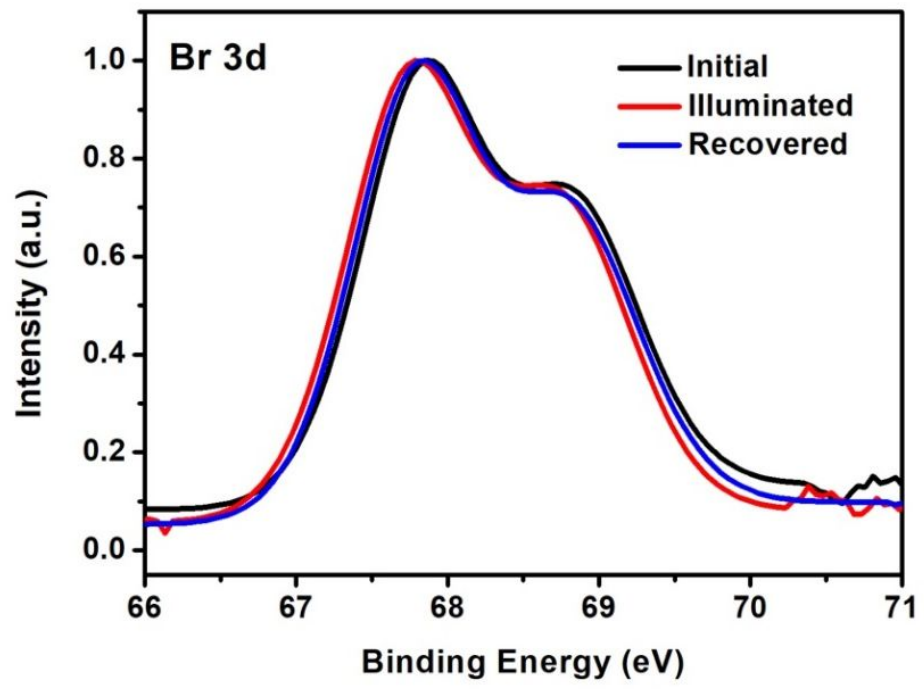

Figure S9. Br 3d XPS spectra of the initial, illuminated, and recovered $\mathrm{CsPbBr}_{1.2} \mathrm{I}_{1.8}$ PQDs. 


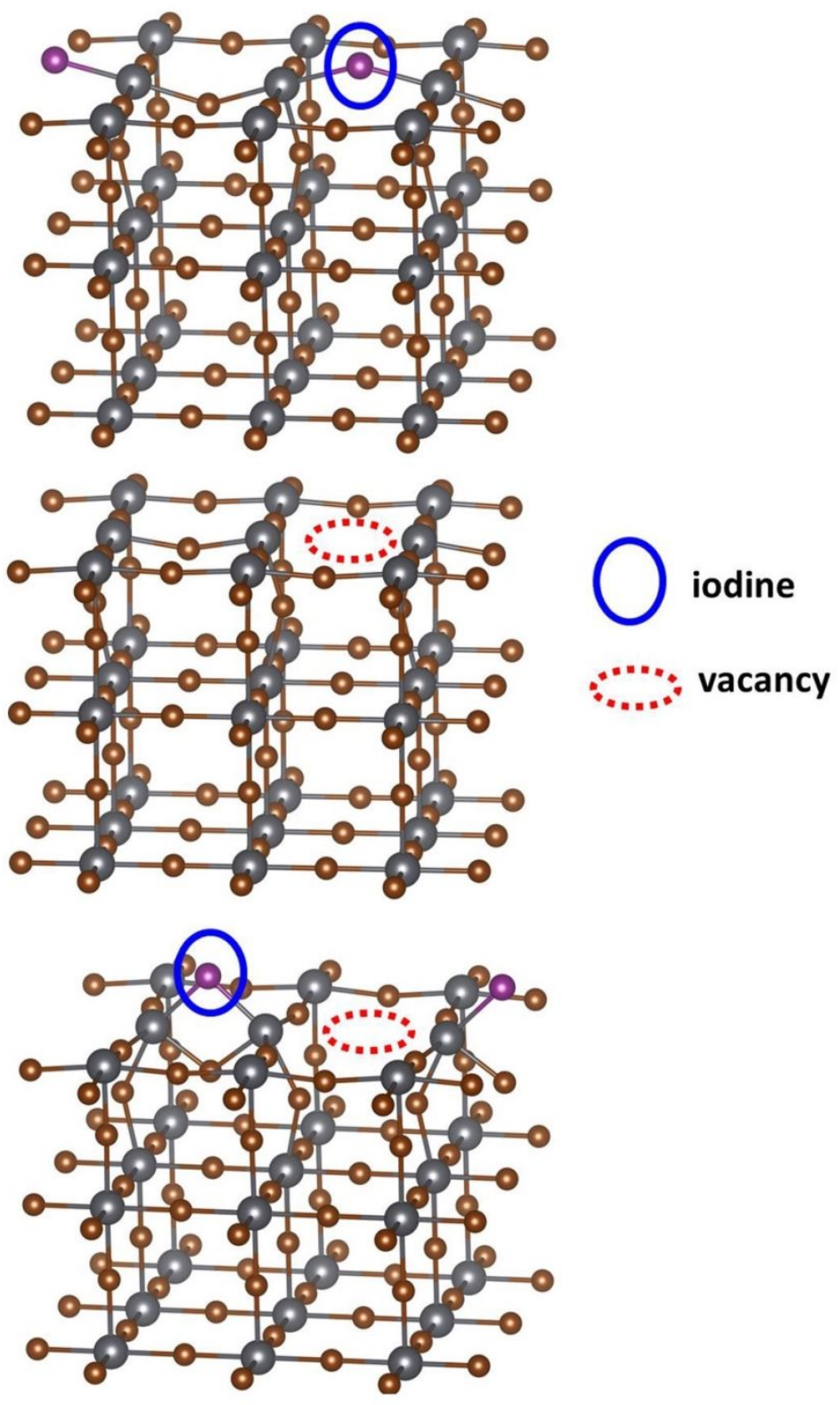

Figure S10. Optimized geometries (side view) of the Model 1, Model 2 and Model 3 (cesium atoms are not shown). 


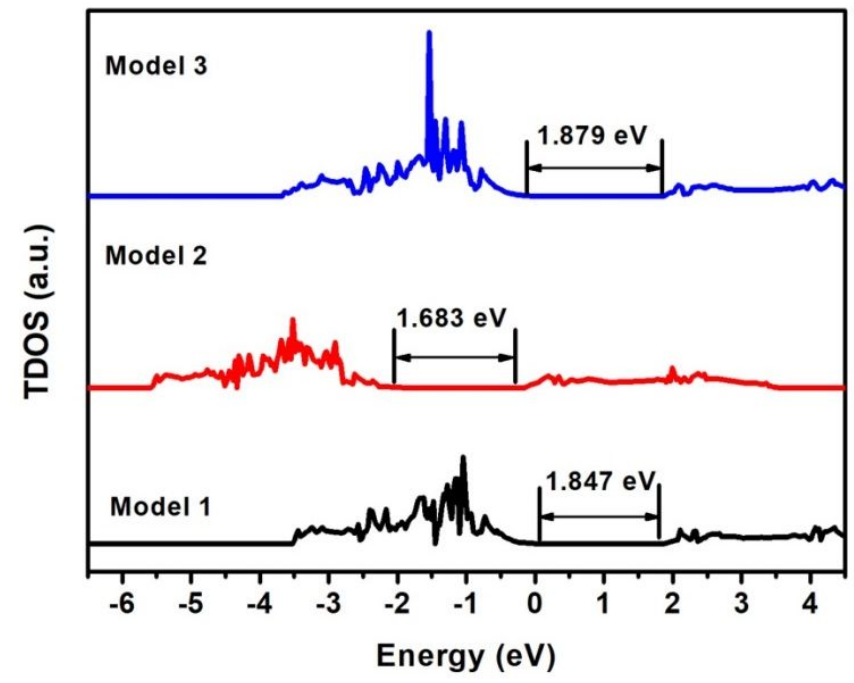

Figure S11. TDOS spectra of the Model 1, Model 2 and Model 3.

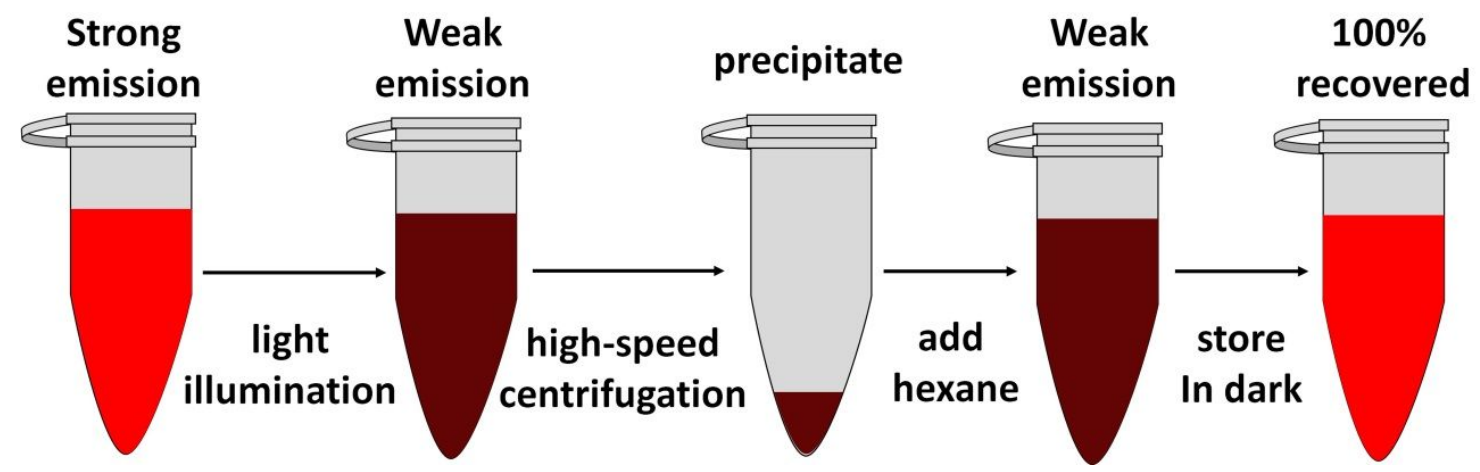

Figure S12. Schematic illustration of the experiment. 
Table S1. Emission recovery of $\mathrm{CsPbBr}_{1.2} \mathrm{I}_{1.8} \mathrm{PQDs}$ starting from different remaining $\mathrm{PL}$ intensities

\begin{tabular}{cccccc}
\hline Remaining PL intensity of the original value (\%) & 80 & 60 & 40 & 20 & 1 \\
\hline Illumination time (min) & 0.5 & 1.5 & 3.2 & 8 & 100 \\
\hline Time to complete recovery (h) & 0.5 & 0.8 & 1.5 & 2.5 & 6 \\
\hline Final PL intensity in respect to original value (\%) & 108 & 107 & 107 & 103 & 98 \\
\hline
\end{tabular}

Table S2. DFT calculation results for the Model 1 and $\mathrm{CsPbl}_{3} \mathrm{PQDs}$

\begin{tabular}{ccc}
\hline Energy & Model 1 & $\mathrm{CsPbl}_{3}$ \\
\hline Surface energy $(\mathrm{eV})$ & -159.561 & -141.124 \\
\hline Iodide ion energy $(\mathrm{eV})$ & -0.26431 & -0.26431 \\
\hline Final energy $(\mathrm{eV})$ & -163.149 & -144.121 \\
\hline Adsorption energy $(\mathrm{eV})$ & -3.3236 & -2.7329 \\
\hline Gibbs free energy change $(\mathrm{eV})$ & -3.291 & -2.698 \\
\hline
\end{tabular}

